\title{
Optimization of STEM Moiré analysis for Two-Dimensional Strain Characterization
}

Mu-Tung Chang ${ }^{1}$, Ren-Fong Cai ${ }^{2}$, ShihYi Liu ${ }^{2}$ and Shen-Chuan $\mathrm{Lo}^{2}$

${ }^{1}$ Material and Chemical Research Laboratories, Industrial Technology Research Institute, Hsinchu 31040, Taiwan, Hsinchu, Taiwan (Republic of China), ${ }^{2}$ Material and Chemical Research Laboratories, Industrial Technology Research Institute, Hsinchu 31040, Taiwan, United States

Group III-V compound semiconductors and silicon germanium ( $\mathrm{SiGe}$ ) materials have many developments in optoelectronics, semiconductors, and industrial applications, including high-speed components, highpower components, and optoelectronic components. The combination of heterogeneous interfaces is also a very interest and important research topic. The modulation of its strain and stress plays a critical role in the advanced and valuable device. In order to understand the relationship between its microstructure and strain, a complete analysis platform needs to be established to provide industry a clearer understanding of the application. Many methods of measuring strain-stress have been developed, including Peak-Pairs Analysis (PPA), Geometric Phase Analysis (GPA), Nano Beam Diffraction (NBD) and Scanning transmission electron microscopy (STEM) Moiré interference images. Among these methods, the STEM Moiré interference can be performed with a wide range and high precision stress analysis. In this research, we try to optimize the image capture method of STEM Moiré. Traditionally, the interference images were captured through ADF detectors at a fixed magnification in the STEM mode. In this image capture mode, an interference image with high signal-to-noise ratio often takes 30 seconds to capture. We hope to shorten the overall acquisition time without reducing the image quality. The introduction of such a concept can reduce the strain calculation error caused by sample drift. Using the energy spectrum imaging function in the GMS software developed by Gatan, we can freely choose the analysis area. When we choose a smaller analysis area, we can get the stress distribution value of the small area in a short analysis time. We can usually control the overall image capture time within 5 seconds. This analysis method can effectively reduce the stress deviation caused by sample drift. Although the analyzed area becomes smaller, we can still get a wide range of strain analysis results through multi-point nano-area strain analysis. Such stress analysis concepts and methods are expected to be applied to more advanced semiconductor devices. 


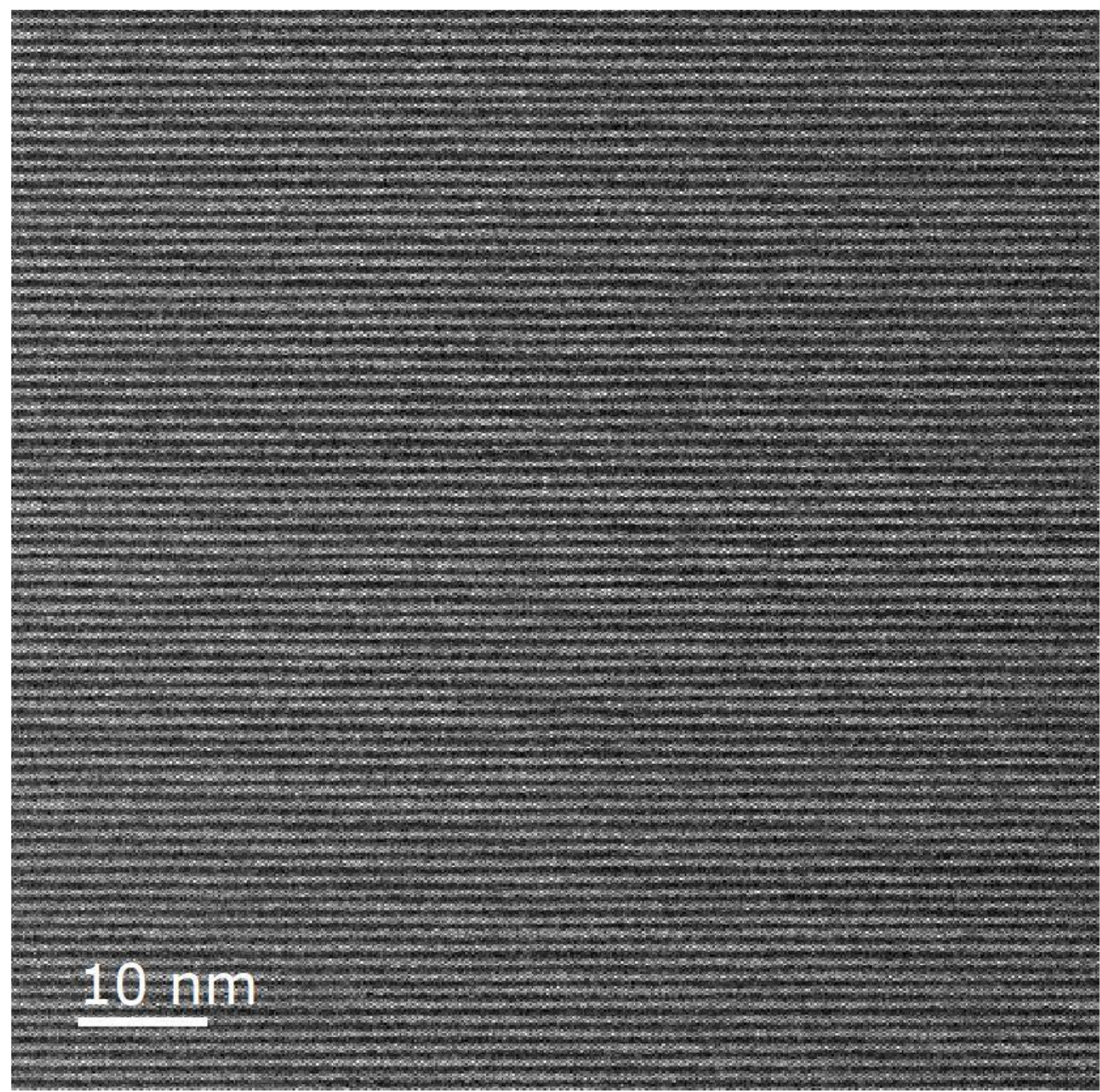

Figure 1. Fig. 1 the traditional interference image was captured through ADF detectors at a fixed magnification in the STEM mode 


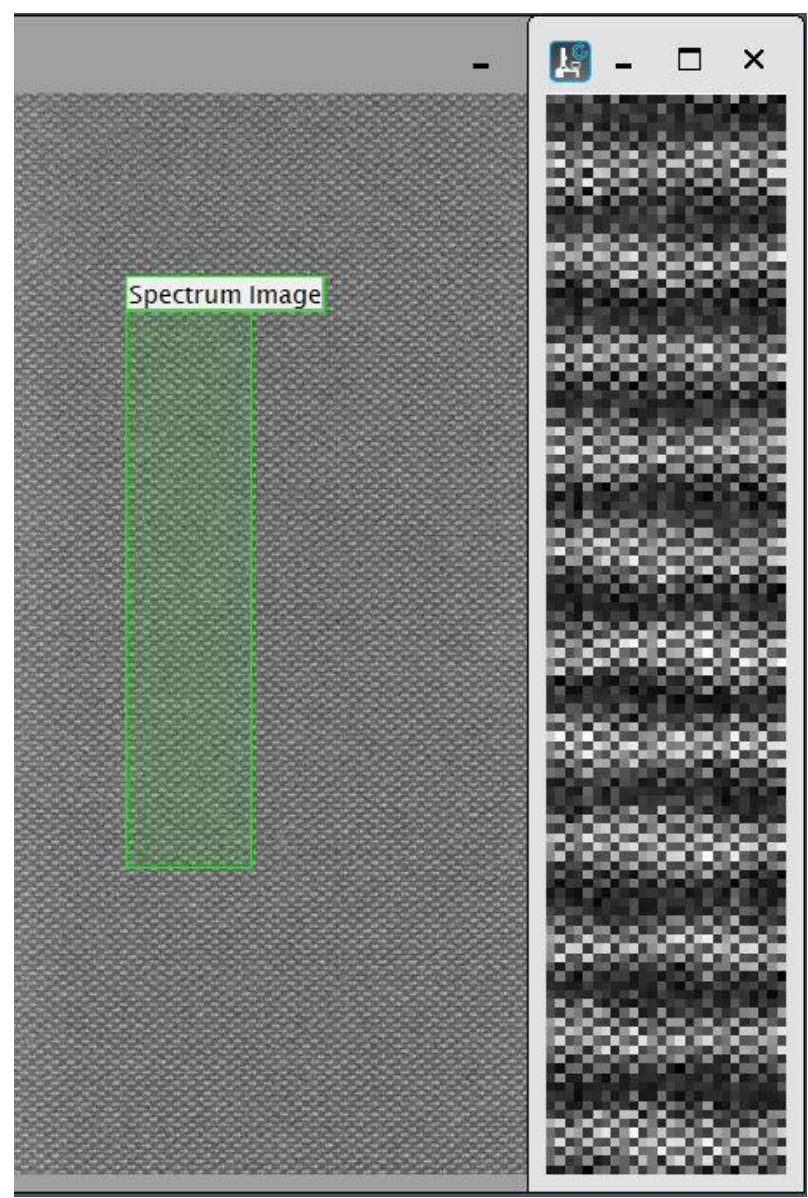

Figure 2. Fig. 2 the interference image with shorter capture time using the energy spectrum imaging function in the GMS software

\section{References}

1. Akimitsu Ishizuka, Martin Hytch, Kazuo Ishizuka, "STEM moiré analysis for 2D strain measurements", Microscopy, Volume 66, Issue 3, June 2017, Pages 217-221 